\title{
Nonlinear instability of baroclinic atmosphere with reference to planetary scale disturbances
}

\author{
I. A. Pisnichenko \\ Center for Weather Forecasting and Climate Studies/Brazilian National Institute for Space Research, Brazil \\ on leave from A. M. Obukhov Institute of Atmospheric Physics, Russian Academy of Sciences, Moscow, Russia
}

Received: 27 May 2003 - Revised: 12 July 2004 - Accepted: 24 July 2004 - Published: 2 August 2004

\begin{abstract}
In this paper we investigate the stability of zonal flow in a baroclinic atmosphere with respect to finiteamplitude planetary-scale disturbances by applying Arnold's method. Specifically, we examine the sign of the second variation of a conserved functional for the case of a polytropic atmosphere (i.e. one with a linear lapse rate) and with a linear profile of zonal wind. Sufficient stability conditions for an infinite atmosphere (i.e. with a temperature lapse rate equal to zero) are satisfied only for an atmosphere in solid body rotation. For a polytropic atmosphere of finite extent (a lapse rate is not equal zero) the sufficient conditions of stability can be satisfied if a lid is placed below $\min \left(Z_{\max }\right.$, polytropic atmospheric height). The dependence of height $Z_{\max }$ on values of the vertical gradient of the zonal wind and the zonal temperature distribution is calculated.
\end{abstract}

\section{Introduction}

Ultra-long atmospheric waves play a significant role in the formation of weather and climate regimes. The longest waves dominate the spectral distribution of kinetic and available potential energy. The ultra-long waves are responsible for both energy transfer through the spectrum (Baines, 1976; Starr, 1968) and for heat transfer from the lowest layer of the troposphere to the stratosphere (Charney and Drazin, 1961; McNulty, 1976). The first three spherical harmonic components $\left(\psi_{1}^{0}, \psi_{1}^{1}, \psi_{1}^{-1}\right)$ are directly connected with the angular momentum of the Earth (Barnes et al, 1983; Pisnichenko, 1990). There is a large correlation between events of blocking formation and the values of amplitudes and phases of stationary spherical harmonics with $m=2,3$ in the geopotential field (Kurbatkin, 1970; Bengtsson, 1981). Planetary waves are also responsible for low-frequency atmospheric variations and, as a consequence, for the manner of the temporal evolution of stochastic atmospheric regimes (James and

Correspondence to: I. A. Pisnichenko

(pisnitch@cptec.inpe.br)
James, 1992; Kurgansky et al, 1996). For these reasons, investigation of peculiarities in the behaviour of ultra-long waves is important for undestanding of climate processes and for the construction of climate simulation models.

Some of the principal properties of ultra-long waves are related to the stability of the atmospheric zonal flow. The growth rates and the height distribution of the amplitudes of unstable ultra-long waves are central characteristics that, to a great extent, define the dynamics of the large-scale atmospheric circulation.

In Lynch (1979), the instability of zonal flow with respect to ultra-long waves was studied by means of linearised planetary geostrophic equations of type II. These equations describing planetary-scale disturbances were first proposed by Burger (1958) and were later used in the works of WiinNielsen (1961), Phillips (1963), Pisnichenko (1980, 1983), and others. Important insights into the dependence of the growth rate of ultra-long waves on the wind shear and the coefficient of static stability were obtained using this linear approach.

It is also interesting to solve the problem in its nonlinear formulation and to study the role of nonlinearity on zonal flow stability. Beginning with Arnol'd (1965), the method of construction and investigation of a conserved functional from instantaneous states of hydrodynamical fields has been widely used in the study of fluid flow stability. Application of this method to determine the stability of three-dimensional baroclinic flows in meteorological models first appeared in Dikiy (1965) and Dikiy and Kurgansky (1971), and later in studies such as McIntyre and Shepherd (1987); Shepherd (1989); Mu and Shepherd (1994); Yongming and Mu (1996).

This paper addresses zonal flow stability with respect to ultra-long wave disturbances within a fully nonlinear framework. We consider the problem in the approximation of planetary geostrophic equations which are presented in Sect. 2, together with conservation laws for the model in question. In Sect. 3, Bernoulli's theorem is formulated for this system. It is also shown that the stream function of the flow under investigation is fully defined by potential vorticity and potential 
temperature fields. Section 4 is devoted to the calculation of first and second variations of a functional composed of conservation integrals. The conditions of sign definiteness of the second variation define the sufficient conditions of zonal flow stability. The general equation obtained here are applied for the case of polytropic atmospheres in Sect. 5 .

\section{The model equations}

We will consider three-dimensional baroclinic atmospheric flow of planetary scale on the spherical Earth. The equations used are the quasi-geostrophic equations of type II first derived by Burger (1958). For the dry adiabatic case they are written in the form:

$f u=-\frac{1}{\rho a} \frac{\partial p}{\partial \varphi}$

$-f v=-\frac{1}{\rho a \cos \varphi} \frac{\partial p}{\partial \lambda}$

$g=-\frac{1}{\rho} \frac{\partial p}{\partial z}$

$\frac{\partial \rho}{\partial t}+\nabla \cdot \rho \mathbf{v}=0$

$\frac{d s}{d t}=0$

$p=R \rho T$

where $u, v, w$ are the zonal, meridional and vertical components of velocity vector $\mathbf{v} ; p, \rho$, and $s$ are respectively the air pressure, density, and specific entropy of dry air with gas constant $R ; f=2 \omega \sin \varphi$ is the Coriolis parameter; $\omega$ is the angular velocity of the Earth's rotation; $g$ is the gravitational acceleration force for unit mass; and $\lambda, \varphi, z$ are longitude, latitude and height above the surface of a spherical Earth of radius $a$. Differential operators of total derivative $d / d t$ and divergence in spherical coordinates are

$$
\begin{aligned}
& \frac{d}{d t}=\frac{\partial}{\partial t}+\frac{u}{a \cos \varphi} \frac{\partial}{\partial \lambda}+\frac{v}{a} \frac{\partial}{\partial \varphi}+w \frac{\partial}{\partial z} . \\
& \nabla \cdot \mathbf{v}=+\frac{1}{a \cos \varphi} \frac{\partial u}{\partial \lambda}+\frac{1}{a \cos \varphi} \frac{\partial}{\partial \varphi}(\cos \varphi \quad v)+\frac{\partial w}{\partial z}
\end{aligned}
$$

From equations ( $1-6)$, the following conservation laws can be obtained:

a) conservation of the planetary potential vorticity (transposed potential vorticity) of an air parcel (see Appendix for details)

$$
\frac{d}{d t} \frac{f \frac{\partial s}{\partial z}}{\rho}=\frac{d \Omega}{d t}=0
$$

b) globally-integrated conservation of total potential energy

$$
\frac{d}{d t} \iiint_{V} \rho\left(g z+c_{v} T\right) d \tau=\frac{d \Pi}{d t}=0 ;
$$

c) conservation of any globally-integrated mass-weighted function of potential vorticity and potential temperature

$$
\frac{d}{d t} \iiint_{V} \rho \Phi(\Omega, s) d \tau=\frac{d F}{d t}=0,
$$

where $\Phi$ is any function of $\Omega$ and $s$;

d) conservation of transposed angular momentum (the angular momentum of the atmosphere if it were in solid rotation with the earth)

$\frac{d}{d t} \iiint_{V} \omega a^{2} \cos ^{2} \varphi \rho d \tau=\frac{d M}{d t}=0$.

e) conservation of any surface-integrated $f$-weighted function of entropy

$\frac{d}{d t} \iint_{S} f G(s) d \sigma=\frac{d P}{d t}=0$,

where $G$ is an arbitrary function of specific entropy and the integral is calculated over the plane Earth's surface ( $w$ should be equal to zero at the Earth's surface).

\section{Bernoulli's theorem}

We will investigate the stability of atmospheric zonal flow according to the scheme described by Dikiy (1965) and Dikiy and Kurgansky (1971). We begin by showing that a steady flow $(\partial / \partial t=0)$ described by Eqs. (1-6) satisfies Bernoulli's theorem. To prove this, multiply Eqs. (1), (2), and (3) by $a d \varphi, a \cos \varphi d \lambda$ and $d z$, respectively, and add. We obtain:

$f(u a d \varphi-v a \cos \varphi d \lambda)+g d z=-\frac{1}{\rho} d p$.

For a streamline,

$$
\frac{a \cos \varphi d \lambda}{u}=\frac{a d \varphi}{v}=\frac{d z}{w},
$$

so the parenthetical term in Eq. (12) drops out along a streamline, leaving Bernoulli's theorem:

$d g z=-\frac{1}{\rho} d p$.

Next we show that $d s=0$ along a streamline. For this, multiply Eq. (5) for steady flow by $d t$ and note that $u d t=$ $a \cos \varphi d \lambda, v d t=a d \varphi$, and $w d t=d z$. Then, using the first law of thermodynamics, we obtain $d\left(g z+c_{p} T\right)=0$ or

$g z+c_{p} T=\mathrm{const}$

along a streamline.

Let us assume further that $\nabla s \times \nabla \Omega \neq 0$, so that isentropic and isovorticial surfaces do not touch each other any where in steady flow. One can show that this property will be conserved for all time (Kurgansky, 1993). Thus, every streamline is uniquely determined by $s$ and $\Omega$. That is

$\Psi(\lambda, \varphi, z, t)=g z+c_{p} T=\Psi(s, \Omega)$. 


\section{A conserved functional and its first and second varia- tions}

To investigate the stability of a zonal flow let us compose the functional from the integrals of motion (Eqs. 8-11). This procedure is to some extent similar to finding conditional extremum of a function with the help of a Lagrange multiplier. The integrals of motion that we use here correspond to constraints that set some limitations on possible motions.

$$
\begin{aligned}
& H=\Pi+F+P+q M=\iiint_{V} \rho\left[g z+c_{v} T+\Phi(s, \Omega)+\right. \\
& \left.+q \omega a^{2} \cos ^{2} \varphi\right] d \tau+\iint_{S} f G(s) d \sigma .
\end{aligned}
$$

The temperature $T$ in functional $H$ can be expressed in terms of specific entropy as $T=T^{*} \exp \left(s / c_{v}\right)\left(\rho / \rho_{0}\right)^{\kappa-1}$ where $\kappa=c_{p} / c_{v}$ and $T^{*}$ is a constant having the dimension of temperature. Symbol $q$ corresponds here to any arbitrary constant. Denoting the value of the functional $H$ corresponding to the zonal flow under discussion as $H_{0}$ and expanding the functional $H$ in a Taylor series in the vicinity of $H_{0}$ we write:

$$
H=H_{0}+\left.\delta H\right|_{H_{0}}+\left.\delta^{2} H\right|_{H_{0}}+\ldots
$$

If the first functional variation $\left.\delta H\right|_{H_{0}}$ is equal to zero, then the zonal flow corresponding to the functional value $H_{0}$ is a stationary point in the functional $\{\Omega, s\}$-space. Performing the calculation we will obtain the following expression for the first variation of $H$ :

$$
\begin{aligned}
& \delta H=\iiint_{V}\left[\left(\Psi+\Phi-\Phi_{\Omega} \Omega+q \omega a^{2} \cos ^{2} \varphi\right) \delta \rho\right. \\
& \left.+\rho\left(T+\Phi_{s}-\Phi_{s \Omega} \Omega-\Phi_{\Omega \Omega} \frac{f}{\rho} \frac{\partial \Omega}{\partial z}\right) \delta s\right] d \tau \\
& +\iint_{S} f\left(G_{s}-\Phi_{\Omega}\right) \delta s d \sigma .
\end{aligned}
$$

One can see from Eq. (15) that necessary conditions for $\delta H=0$ are the following

$$
\begin{aligned}
& \Psi+\Phi-\Phi_{\Omega} \Omega+q \omega a^{2} \cos ^{2} \varphi=0 \\
& \Phi_{s}-\Phi_{\Omega s} \Omega-\Phi_{\Omega \Omega} \frac{f}{\rho} \frac{\partial \Omega}{\partial z}+T=0 \\
& G^{\prime}(s)-\left.\Phi_{\Omega}\right|_{z=0}=0
\end{aligned}
$$

Note here that Eq. (17) is a consequence of Eq. (16). To prove this, it suffices to differentiate Eq. (16) with respect to $z$ and then to divide the result obtained by $\partial s / \partial z \neq 0$. If arbitrary functions $\Phi$ and $G$ are chosen to satisfy Eqs. (16) and (18) then for our zonal flow $H_{0}$ will be a stationary point.

For the second variation of $H$ we will have:

$$
\begin{aligned}
& \delta^{2} H=\iiint_{V}\left[\frac{(\kappa-1) c_{p} T}{\rho}(\delta \rho)^{2}+2\left(\kappa T-\Phi_{s \Omega} \Omega\right.\right. \\
& \left.+\Phi_{s}\right) \delta \rho \delta s+\rho \Phi_{\Omega \Omega}(\delta \Omega)^{2}+\rho\left(\Phi_{s s}+\frac{T}{c_{v}}-\Phi_{\Omega s s} \Omega\right. \\
& \left.\left.-\Phi_{\Omega \Omega s} \frac{f}{\rho} \frac{\partial \Omega}{\partial z}\right)(\delta s)^{2}\right] d \tau+\iint_{S}\left(G_{s s}-\Phi_{\Omega s}\right) f(\delta s)^{2} d \sigma .
\end{aligned}
$$

Here we designate $\delta \Omega=f / \rho \delta(\partial s / \partial z)-f / \rho^{2} \partial s / \partial z \delta \rho$, and $\Phi_{\Omega}, \Phi_{s}, \ldots$ as partial derivatives of function $\Phi$ with respect to $\Omega, s$.

Differentiating Eq. (17) with respect to $z$, we find:

$\Phi_{s s}-\Phi_{\Omega s s}-\Phi_{\Omega \Omega s} \frac{f}{\rho} \frac{\partial \Omega}{\partial z}=\frac{1}{s_{z}}\left[\frac{\partial}{\partial z}\left(\Phi_{\Omega \Omega} \frac{f}{\rho} \frac{\partial \Omega}{\partial z}\right)-T_{z}\right]$,

and finally differentiating Eq. (16) with respect to $\phi$ and taking into account Eq. (17), we obtain:

$\Phi_{\Omega \Omega}=\frac{\rho\left(q \omega a^{2} \sin 2 \varphi+T \frac{\partial s}{\partial \varphi}-c_{p} \frac{\partial T}{\partial \varphi}\right)}{(s, \Omega) a f}$.

Here $(s, \Omega)=(\partial s / \partial \varphi \partial \Omega / \partial z-\partial \Omega / \partial \varphi \partial s / \partial z) / a$.

Using Eqs. (17), (20), (21) we can rewrite the second variation of $H$ as:

$$
\begin{aligned}
& \delta^{2} H=\iiint_{V}\left\{\frac{1}{\rho}(\kappa-1) c_{p} T(\delta \rho)^{2}+2\left[(\kappa-1) T+\left(\frac{\partial \Omega}{\partial z}\right)\right.\right. \\
& \left.\cdot \frac{f u+q \omega a \sin 2 \varphi}{(s, \Omega)}\right] \delta \rho \delta s+\frac{\rho^{2}(f u+q \omega a \sin 2 \varphi)}{f(s, \Omega)}(\delta \Omega)^{2}+ \\
& \left.\rho\left[\frac{T}{c_{v}}+\frac{1}{s_{z}}\left[\frac{\partial}{\partial z}\left(\frac{\partial \Omega}{\partial z} \frac{f u+q \omega a \sin 2 \varphi}{(s, \Omega)}\right)-T_{z}\right]\right](\delta s)^{2}\right\} d \tau \\
& +\iint_{S} \rho \frac{\Omega_{\varphi}}{s_{\varphi}} \frac{(f u+q \omega a \sin 2 \varphi)}{(s, \Omega)}(\delta s)^{2} d \sigma .
\end{aligned}
$$

The surface integral expression was obtained by differentiating Eq. (18) with respect to $\varphi$. We also have used here the alternative form of Eq. (1)

$f u=-\frac{c_{p}}{a} \frac{\partial T}{\partial \varphi}+\frac{T}{a} \frac{\partial s}{\partial \varphi}$.

Note that $\delta^{2} H$ represents a functional from two independent functions because $\Omega$ can be expressed through $\rho$ and $s$.

If we consider zonal flow with $\delta H=0$, and if $\delta^{2} H$ is sign-definite at a certain point in functional space of two independent functions, then this point will be a maximum (or minimum) of functional $H$ in this functional space. Level functional surfaces in functional space around this point will be closed and inserted one into another. Thus, in the case of a finite disturbance of the zonal flow, the phase point corresponding to the new disturbed state will shift to one of these surfaces and will stay on it, as $d H / d t=0$. During all next time, the phase point will not strongly deviate from the extremum point corresponding to the initial zonal flow (Arnol'd, 1965).

\section{The case of a polytropic atmosphere}

Let us now consider a polytropic atmosphere $\left(T=T_{0}-\gamma z\right)$. We will assume that the wind changes linearly with a height: $V=V_{0}+\Lambda z$, as is frequently used in zonal flow stability investigations. 
For further calculations it is convenient to express terms entering the functional (22) through $\Lambda, \gamma, T, \rho$.

$\delta^{2} H=\iiint_{V}\left\{\frac{1}{\rho}\left[(\kappa-1) c_{p} T-\frac{\kappa^{2} g\left(f u+q \omega a \sin 2 \varphi\left(\gamma_{a}-\gamma\right)\right.}{\left(\frac{f \Lambda \kappa}{\kappa-1}+\frac{\beta g\left(\gamma_{a}-\gamma\right)}{f \gamma_{a}}\right) \gamma_{a}}\right](\delta \rho)^{2}+\right.$
$+2\left[(\kappa-1) T-\frac{g(f u+q \omega a \sin 2 \varphi)\left(\gamma_{a}+\kappa(\kappa-1)\left(\gamma_{a}-\gamma\right)\right)}{\left(\frac{f \Lambda \kappa}{\kappa-1}+\frac{\beta g\left(\gamma_{a}-\gamma\right)}{f \gamma_{a}}\right) R \gamma_{a}}\right] \delta \rho \delta s+$
$+\rho\left[\frac{T}{c_{v}}+\frac{T \gamma}{c_{p}\left(\gamma_{a}-\gamma\right)}-\frac{f T \Lambda \gamma_{a}}{\left(\frac{f \Lambda \kappa}{\kappa-1}+\frac{\beta g\left(\gamma_{a}-\gamma\right)}{f \gamma_{a}}\right) R\left(\gamma_{a}-\gamma\right)}-\right.$
$\left.-\frac{g(f u+q \omega a \sin 2 \varphi)\left(\gamma_{a}-\gamma\right)}{\left.\left(\frac{f \Lambda \kappa}{\kappa-1}+\frac{\beta g\left(\gamma_{a}-\gamma\right)}{f \gamma_{a}}\right) c_{v}^{2} \gamma_{a}\right]}(\delta s)^{2}\right\} d \tau+$
$+\iint_{S} \frac{\left(f^{2} u_{0}+R T \beta\right)\left(f u_{0}+q \omega a \sin 2 \varphi\right) \rho_{0} \gamma_{a}}{\left.u_{0}-\frac{\Lambda}{\gamma_{0}-\gamma}\right)\left(\frac{f \Lambda \kappa}{\kappa-1}+\frac{\beta g\left(\gamma_{a}-\gamma\right)}{f \gamma_{a}}\right)\left(\gamma_{a}-\gamma\right) R f^{2}}(\delta s)^{2} d \sigma .(24)$

Here we designate $\partial f / a \partial \varphi=\beta$ and have used the following equation:

$$
\begin{aligned}
& \frac{\partial s}{\partial z}=\frac{\left(\gamma_{a}-\gamma\right) c_{p}}{T} ; \quad \Omega=\frac{f\left(\gamma_{a}-\gamma\right) c_{p}}{\rho T} ; \\
& \Omega_{z}=\frac{\Omega g}{R T} ; \quad \frac{\partial s}{a \partial \varphi}=\left(\frac{f u}{T}-\frac{f \Lambda}{\gamma_{a}-\gamma}\right) \frac{\gamma_{a}-\gamma}{\gamma_{a}} ; \\
& \frac{\partial \Omega}{a \partial \varphi}=\frac{\Omega}{T}\left(\frac{f u}{R}+\frac{T \beta}{f}\right) ; \delta \Omega=-\frac{\Omega}{c_{v} \rho}\left(\rho \delta s+c_{p} \delta \rho\right) ; \\
& (s, \Omega)=-\frac{\Omega}{T}\left[\frac{f \Lambda \kappa}{\kappa-1}+\frac{\beta g\left(\gamma_{a}-\gamma\right)}{f \gamma_{a}}\right] .
\end{aligned}
$$

Let $u_{0}=-q a \cos \varphi$. This means that the wind on the Earth's surface represents solid body rotation. The intensity and direction of the wind is defined by the arbitrary parameter $q$. In this case, the surface integral in Eq. (24) is zero, and the sufficient conditions required for the volume integral part of $\delta^{2} H$ to be sign-definite quadratic form, are also sufficient conditions for stability of the zonal flow with respect to planetary scale disturbances.

The second variation $\delta^{2} H$ will be sign-definite if:

$$
\begin{aligned}
& \frac{1}{\rho}\left[(\kappa-1) c_{p} T-\frac{\kappa^{2} g(f u+q \omega a \sin 2 \varphi)\left(\gamma_{a}-\gamma\right)}{\left(\frac{f \Lambda \kappa}{\kappa-1}+\frac{\beta g\left(\gamma_{a}-\gamma\right)}{f \gamma_{a}}\right) \gamma_{a}}\right] \\
& \cdot \rho\left[\frac{T}{c_{v}}+\frac{T \gamma}{c_{p}\left(\gamma_{a}-\gamma\right)}-\right. \\
& \left.-\frac{f T \Lambda \gamma_{a}}{\left(\frac{f \Lambda \kappa}{\kappa-1}+\frac{\beta g\left(\gamma_{a}-\gamma\right)}{f \gamma_{a}}\right)\left(\gamma_{a}-\gamma\right) R}-\frac{g(f u+q \omega a \sin 2 \varphi)\left(\gamma_{a}-\gamma\right)}{\left(\frac{f \Lambda \kappa}{\kappa-1}+\frac{\beta g\left(\gamma_{a}-\gamma\right)}{f \gamma_{a}}\right) c_{v}^{2} \gamma_{a}}\right] \\
& -\left[\begin{array}{c}
\left.(\kappa-1) T-\frac{g(f u+q \omega a \sin 2 \varphi)\left(\gamma_{a}+\kappa(\kappa-1)\left(\gamma_{a}-\gamma\right)\right)}{\left(\frac{f \Lambda \kappa}{\kappa-1}+\frac{\beta g\left(\gamma_{a}-\gamma\right)}{f \gamma_{a}}\right) R \gamma_{a}}\right]^{2}>0 .(25)
\end{array}\right.
\end{aligned}
$$

Substituting values for $T=T_{0}-\gamma z$ and for $u=u_{0}+\Lambda z$ we can rewrite it as

$$
\begin{aligned}
& \left(\frac{\kappa f \Lambda}{\kappa-1}+\frac{\beta g\left(\gamma_{a}-\gamma\right)}{f \gamma_{a}}\right)^{-2} \cdot \\
& \left\{z ^ { 2 } \left[\frac{(\kappa-1) \beta^{2} g^{2} \gamma^{2}\left(\gamma_{a}-\gamma\right)}{f^{2} \gamma_{a}}+\kappa(3-\kappa) \Lambda \beta g \gamma^{2}+\right.\right. \\
& \left.+2 \kappa^{2} f^{2} \Lambda^{2} \gamma_{a} \gamma-\kappa(2-\kappa) \Lambda \beta g \gamma_{a} \gamma-\frac{\kappa^{3} f^{2} \Lambda^{2} \gamma_{a}^{2}}{\kappa-1}\left(2+\frac{1}{\kappa(\kappa-1)}\right)\right]+ \\
& +z T_{0}\left[\frac{2 \kappa^{2} f^{2} \Lambda^{2} \gamma_{a}}{\kappa-1}+\kappa(2-\kappa) \Lambda \beta g \gamma_{a}-\kappa(4-\kappa) \Lambda \beta g \gamma+\right. \\
& \left.+\frac{2(\kappa-1) \beta^{2} g^{2} \gamma\left(\gamma_{a}-\gamma\right)}{f^{2} \gamma_{a}}\right]+ \\
& \left.+\kappa T_{0}^{2} \Lambda \beta g+\frac{(\kappa-1) T_{0}^{2} \beta^{2} g^{2}\left(\gamma_{a}-\gamma\right)}{f^{2} \gamma_{a}}\right\}>0 .
\end{aligned}
$$

For an atmosphere of any vertical extent this inequality will be satisfied everywhere only when $\Lambda=0$, (because at the pole the term in rectangular parenthesis at $z^{2}$ will be negative for any $\Lambda$ ), that is, when the whole atmosphere rotates as a solid body (note that $u_{0}=-q a \cos \varphi$ ).

For the case of isothermal stratification Eq. (26) will take the form

$$
\begin{aligned}
& \left(\frac{\kappa f \Lambda}{\kappa-1}+\frac{\beta g}{f}\right)^{-2} \cdot \\
& \left\{-\frac{\kappa^{2} f^{2} \Lambda^{2} \gamma_{a}^{2}\left[(\kappa-1)^{2}+\kappa^{2}\right]}{(\kappa-1)^{2}} z^{2}+\kappa T_{0} \Lambda \gamma_{a}\right. \\
& {\left[\frac{2 f^{2} \kappa \Lambda}{(\kappa-1)}+(2-\kappa) \beta g\right] z+} \\
& \left.+\kappa T_{0}^{2} \Lambda \beta g+\frac{(\kappa-1) T_{0}^{2} \beta^{2} g^{2}}{f^{2}}\right\}>0 .
\end{aligned}
$$

Hence it follows that Eq. (27) is satisfied everywhere only for the trivial case of $\Lambda=0$. The quadratic form $\delta^{2} H$ for an isothermal atmosphere will be sign-indefinite for an atmosphere of any vertical extent, when

$$
\begin{aligned}
& \frac{g^{2} T^{2} \Lambda^{2}}{c_{v}^{2}}\left[\beta g(2-\kappa)+\frac{2 f^{2} \kappa \Lambda}{(\kappa-1)}\right]^{2}+ \\
& +\frac{4 f^{2} g^{2} \Lambda^{2}\left[(\kappa-1)^{2}+\kappa^{2}\right]}{c_{v}^{2}(\kappa-1)^{2}}\left[\frac{g^{2}}{f^{2}}(\kappa-1) T^{2} \beta^{2}+\kappa \beta g T^{2} \Lambda\right]<0 .
\end{aligned}
$$

It can be seen that for the whole atmosphere or a hemisphere this cannot be satisfied because it requires an infinite value of negative wind shift at the equator ( $f \rightarrow 0$ at the equator). Therefore, to draw rigourous conclusions about the stability or instability of the zonal flow to planetary-scale disturbances in an isothermal atmosphere, it is necessary to investigate the problem further: to solve the initial value problem or to prove Chetaev's theorem of instability for a continuous medium (Chetaev, 1990). 


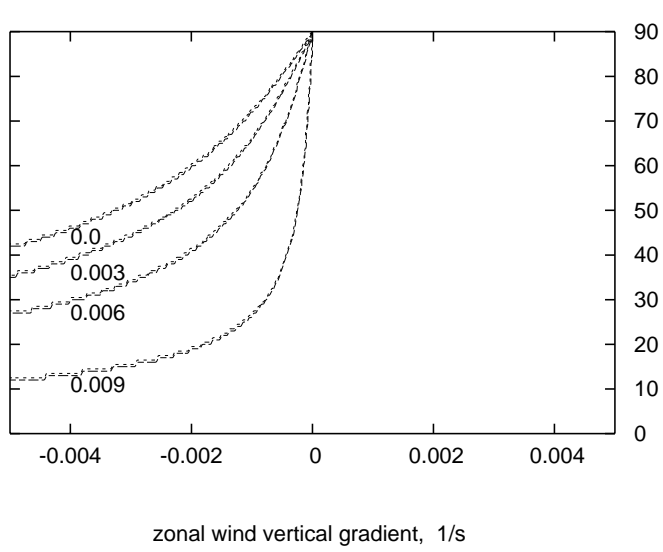

Fig. 1. The areas of stability for a finite atmosphere are located to the right of the curves. The curves marked from top to bottom correspond to lapse rates of $\gamma=0 \mathrm{~K} / \mathrm{m}, 0.003 \mathrm{~K} / \mathrm{m}, 0.006 \mathrm{~K} / \mathrm{m}$, $0.009 \mathrm{~K} / \mathrm{m}$.

On the other hand, the quadratic form $\delta^{2} H$ can be signdefinite for an atmosphere of finite height. For this, it is necessary that one of the roots of Eq. (26) be positive and the other be non-positive, and a lid has to be placed at a height that is equal to or less then the positive root value. Hence the $\delta^{2} H$ will be sign-definite for a finite isothermal atmosphere if

$\Lambda>-\frac{g \beta(\kappa-1)}{f^{2} \kappa}$

and the atmosphere is capped by a lid at positive height $Z_{0} \leq$ $Z_{\max }$ where

$Z_{\max }=$

$\frac{T\left(\frac{\beta g(2-\kappa)}{\kappa}+\frac{2 f^{2} \Lambda}{\kappa-1}+\sqrt{\frac{\beta^{2} g^{2}(\kappa+3)}{\kappa-1}+\frac{4 f^{2} \beta g\left(\kappa^{2}+\kappa-1\right) \Lambda}{\kappa(\kappa-1)^{2}}+\frac{4 f^{4} \Lambda^{2}}{(\kappa-1)^{2}}}\right)}{\frac{2 f^{2} \gamma_{a} \Lambda\left[(\kappa-1)^{2}+\kappa^{2}\right]}{(\kappa-1)^{2}}}$.

Because the zonal velocity changes quasi-linearly with height in free atmosphere, it can be expected that velopause position will be close to $Z_{\max }$. In Fig. 1, the area of stability corresponding to isothermal stratification is situated to the right of the curve marked as 0.0 .

Figure 2 shows the dependence of $Z_{\max }$ on $\varphi$ and $\Lambda$ for isothermal stratification when $\Lambda=$ const in the whole atmosphere, and $T$ is expressed using the thermal wind equation as $T=T_{\mathrm{eq}} e^{D \cos \varphi}$. Here the following designation are used: $D=2 \omega \Lambda / g, T_{\text {eq }}$ is a surface temperature on the equator.

Figure 3 presents the dependence of $Z_{\max }$ on latitude, $\varphi$, and the equator-to-pole temperature contrast, $\Delta T$. Specifically, we model the surface temperature as $T=T_{p}+$ $\Delta T \cos \varphi$, where $T_{p}$ is the surface temperature at the pole and $\Delta T=T_{\mathrm{eq}}-T_{p}$.

In the general case of a polytropic finite atmosphere, the area of stability decreases with increasing lapse rate $\gamma$ (Fig. 1). In the model used, $\gamma$ does not depend on latitude
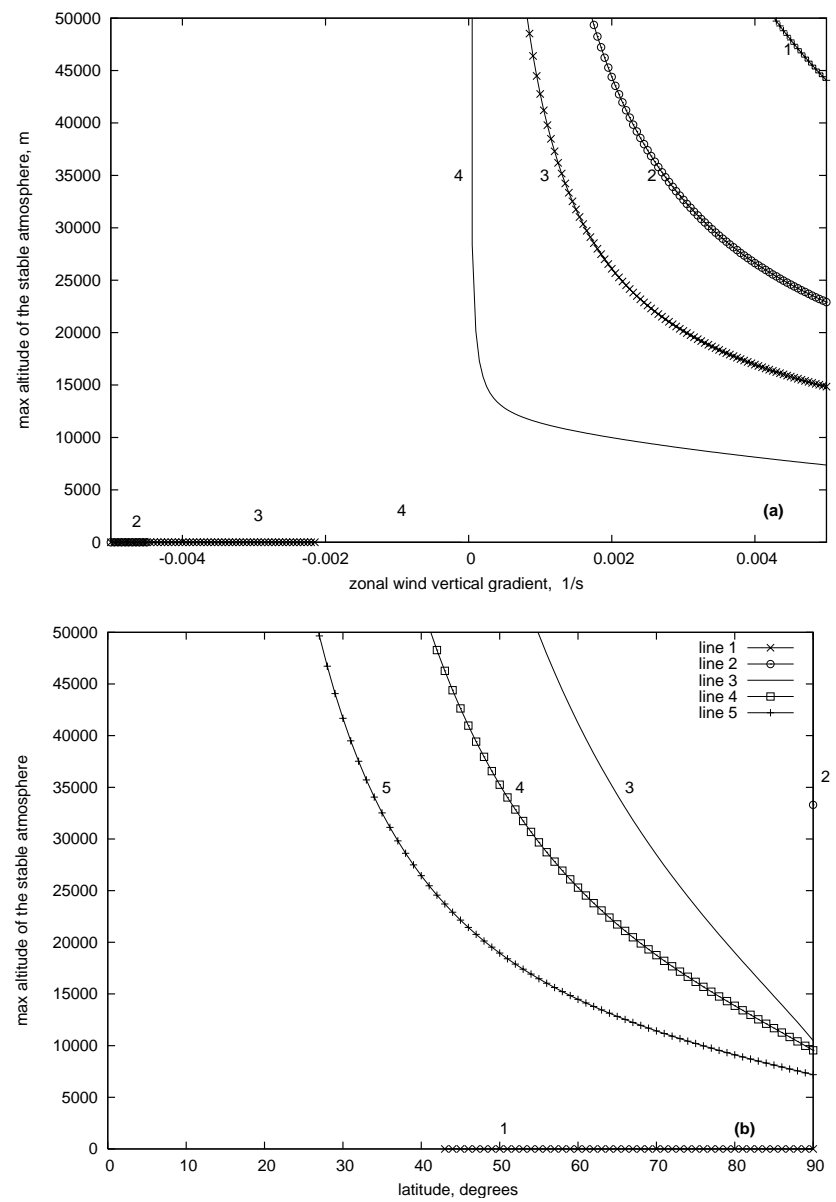

Fig. 2. Dependence of $Z_{\max }$ in a vertically isothermal atmosphere $(\gamma=0)$ on the zonal wind vertical gradient $\Lambda$ (a) and latitude $\varphi$ (b) for the case when $\Lambda$ is constant in the whole atmosphere. In (a), curves $1-4$ correspond to latitudes $30^{\circ}, 45^{\circ}, 60^{\circ}$, and $90^{\circ}$. In (b), curves $1-5$ correspond to $\Lambda=-0.005 \mathrm{~s}^{-1}, 0 \mathrm{~s}^{-1}, 0.001 \mathrm{~s}^{-1}$, $0.002 \mathrm{~s}^{-1}$, and $0.005 \mathrm{~s}^{-1}$.

$\varphi$. To show this, it suffices to differentiate the momentum equation (23) with respect to $z$ :

$\frac{f}{c_{p}} \frac{\partial u}{\partial z}=-\frac{c_{p}}{a} \frac{\partial^{2} T}{\partial z \partial \varphi}+\frac{T}{a} \frac{\partial^{2} s}{\partial z \partial \varphi}+\frac{1}{a} \frac{\partial s}{\partial \varphi} \frac{\partial T}{\partial z}$

Using Eq. (23) and the thermal wind equation

$f \Lambda=-\frac{\gamma f u}{T}-\frac{c_{p} \gamma_{a}}{T a} \frac{\partial T}{\partial \varphi}$

one can express the meridional gradient of entropy as

$\frac{1}{a} \frac{\partial s}{\partial \varphi}=\left[\frac{f u}{T}-\frac{f \Lambda}{\gamma_{a}-\gamma}\right] \frac{\gamma_{a}-\gamma}{\gamma_{a}}$.

Substituting this result into Eq. (29) we conclude that $\partial^{2} T / \partial z \partial \varphi=\partial \gamma / \partial \varphi=0$.

The maximum height of a polytropic stable finite atmosphere model is $\min \left(T_{0} / \gamma, Z_{\max }\right)$. $Z_{\max }$ for this case depends on $\varphi, \Lambda, \gamma$, and $u_{0}=\alpha \cos \varphi$. In Figs. 4, 5, 6 the 

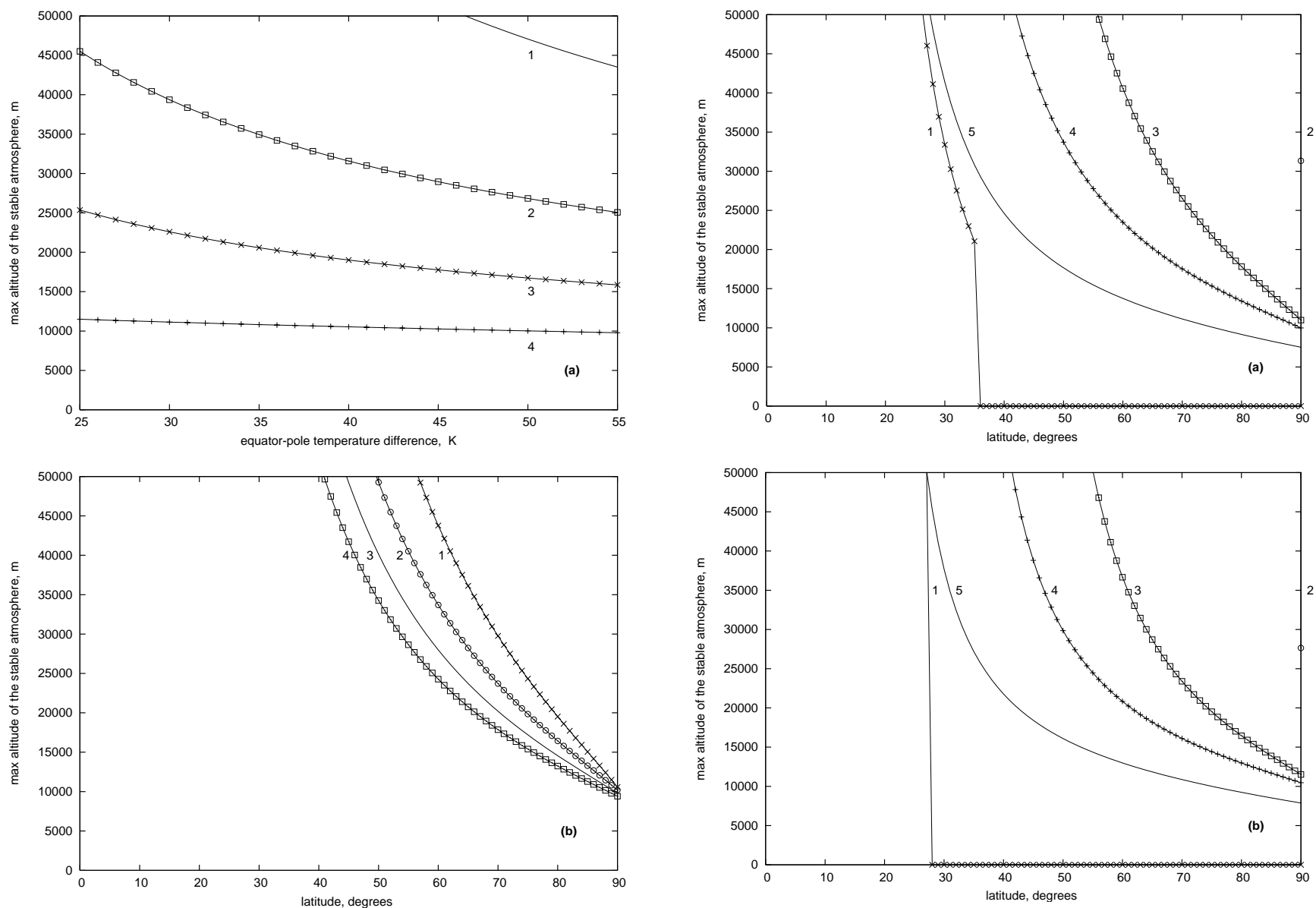

Fig. 3. Dependence of $Z_{\max }$ on the equator-to-pole temperature contrast, $\Delta T$ (a), and on latitude, $\varphi(\mathbf{b})$. The meridional surface temperature distribution is modelled as $T=T_{p}+\Delta T \cos \varphi$, and vertical temperature gradient is constant $(\gamma=0)$. In (a), curves 1-4 correspond to latitudes $45^{\circ}, 60^{\circ}, 75^{\circ}$, and $90^{\circ}$. In (b), curves 1-3 correspond to $\Delta T=25 \mathrm{~K}, 35 \mathrm{~K}, 45 \mathrm{~K}$, and $55 \mathrm{~K}$.

dependence of $Z_{\max }$ on these parameters is presented. Results depend weakly on $\alpha$. Here the calculations were made for $\alpha=5 \mathrm{~m} / \mathrm{s}$. For the calculations with $\Lambda$ which do not depend on latitude, to satisfy the thermal wind equation we modelled surface temperature for the flow of interest as $T_{0}=$ $T_{\mathrm{eq}} \exp \{B(\cos \varphi-1)\}+2 A / B^{2}(1+B) \exp \{B(\cos \varphi-1)\}-$ $2 A / B^{2}(1+B \cos \varphi)$, where $A=\gamma \omega \alpha / a g$, and $B=$ $2 \omega \Lambda a / g$.

In these figures one can see that the rate of the change of $Z_{\max }$ with latitude noticeably depends both on $\Lambda$ and $\gamma$ (Fig. 4). However the minimum height of $Z_{\max }$ on a pole does not strongly vary and is equal to approximately $8-9 \mathrm{~km}$ (Fig. 5b). The dependence of $Z_{\max }$ on the pole-equator temperature difference $\Delta T$, latitude $\varphi$ and temperature gradient $\gamma$ shows that the area of stability strongly diminishes under the approximation $\gamma$ equals $\gamma_{a}$ (Fig. 6a).

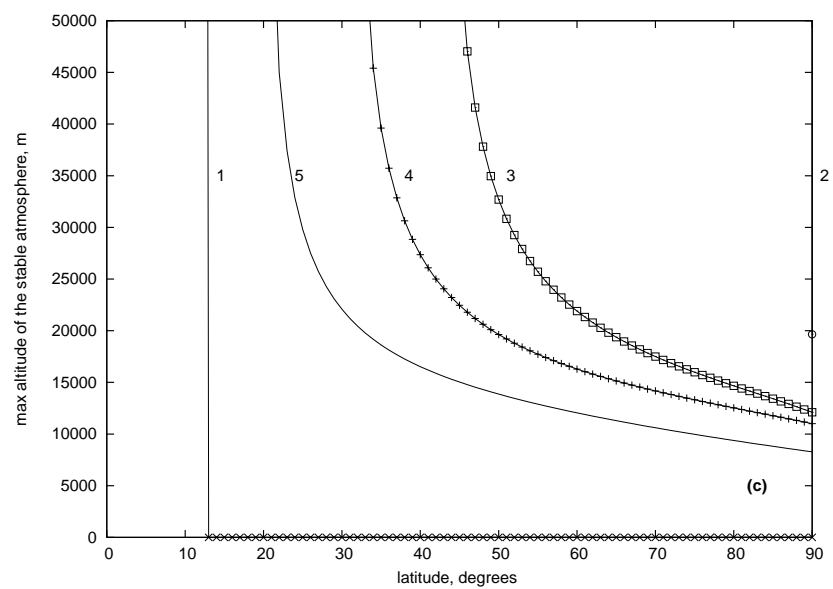

Fig. 4. Dependence of the maximum height $Z_{\max }$ of a finite stable atmosphere on latitude $\varphi$ for three values of $\gamma: 0.003 \mathrm{~K} / \mathrm{m}$ - in (a), $0.006 \mathrm{~K} / \mathrm{m}$ - in (b), and $0.009 \mathrm{~K} / \mathrm{m}$ - (c). The curves labelled $1-5$ correspond to constant values of $\Lambda=-0.005 \mathrm{~s}^{-1}, 0 \mathrm{~s}^{-1}$, $0.001 \mathrm{~s}^{-1}, 0.002 \mathrm{~s}^{-1}$, and $0.005 \mathrm{~s}^{-1}$.

\section{Discussion and conclusions}

In this work we have studied the stability of zonal flow in a baroclinic atmosphere with respect to ultra-long waves. We have considered finite amplitude disturbances and applied Arnol'd's method to type II geostrophic equations. 

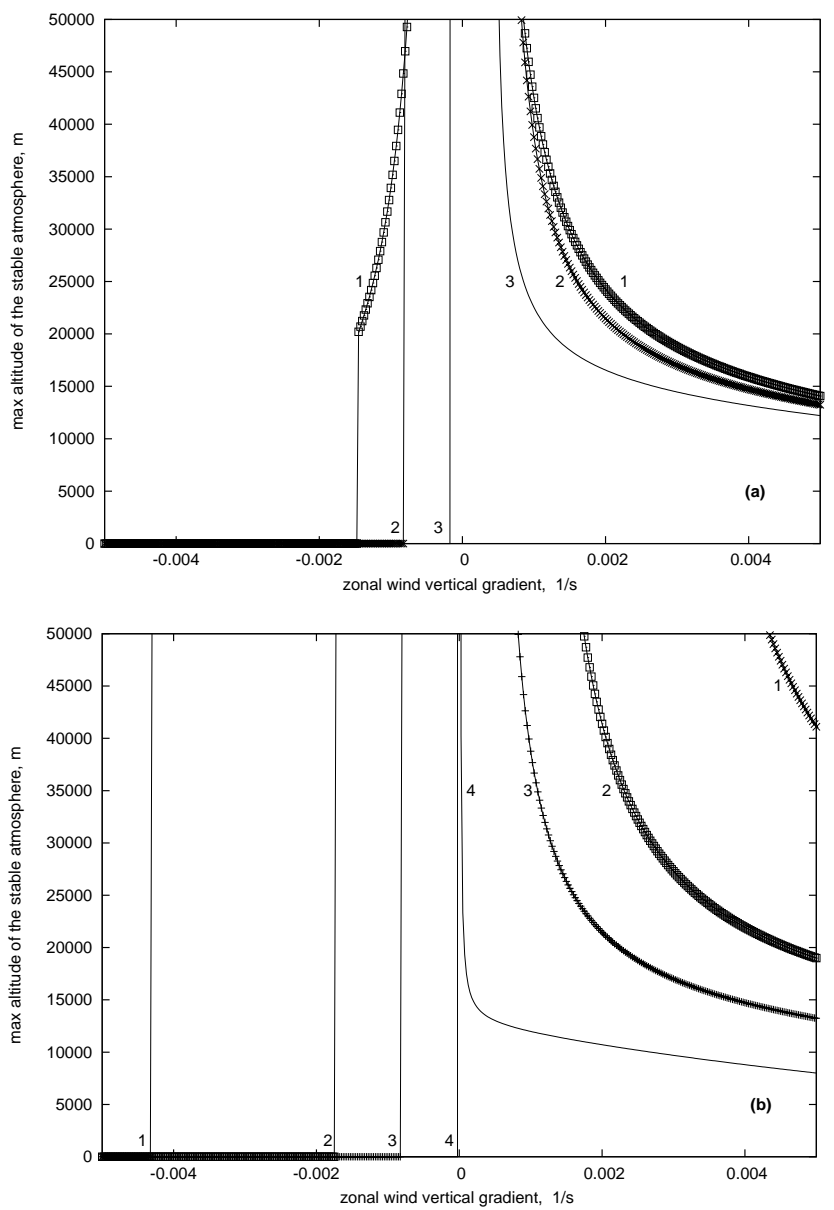

Fig. 5. Dependence of the maximum height of a finite stable atmosphere $Z_{\max }$ on the zonal wind vertical gradient $\Lambda$. In (a) for latitude $\varphi=60^{\circ}$ the curves marked by numbers correspond to: ' 1 ' to $\gamma=0.003 \mathrm{~K} / \mathrm{m}$, '2' $-\gamma=0.006 \mathrm{~K} / \mathrm{m}$, ' 3 ' $-\gamma=0.009 \mathrm{~K} / \mathrm{m}$; on (b) for the stratification with $\gamma=0.006 \mathrm{~K} / \mathrm{m}$ the curves marked by numbers corresponding: ' 1 ' to $\varphi=30^{\circ}$, '2' $-\varphi=45^{\circ}$, ' 3 ' $\varphi=60^{\circ},{ }^{\prime} 4^{\prime}-\varphi=90^{\circ}$. The calculations correspond to the case of $\Lambda=$ const.

For the case of surface wind in solid body rotation, the stability of the zonal flow depends on the sign-definiteness only of that part the second variation of a functional which is represented by the three-dimensional integral. Zonal flow stability was examined directly for the case when the zonal wind changes linearly with height in a polytropic atmosphere.

In an isothermal atmosphere of infinite height, the zonal flow is stable only if the whole atmosphere rotates as a solid body. In an atmosphere bounded by an upper lid, the zonal flow can be stable when vertical shear in zonal wind exists.

For a negative value of the zonal wind vertical gradient $(\partial V / \partial z<0)$, that is, when surface air temperatures increase from equator to pole, the atmosphere is always unstable. For a positive value of $\partial V / \partial z$, stability depends on the height at which the lid is placed. The height of the lid in turn depends on $\partial V / \partial z$ and latitude, and decreases when $\partial V / \partial z$ and latitude increase. For stability, the minimum height at which the lid would have to be placed at the pole is $8-9 \mathrm{~km}$ for the case of the observed values of vertical shear.
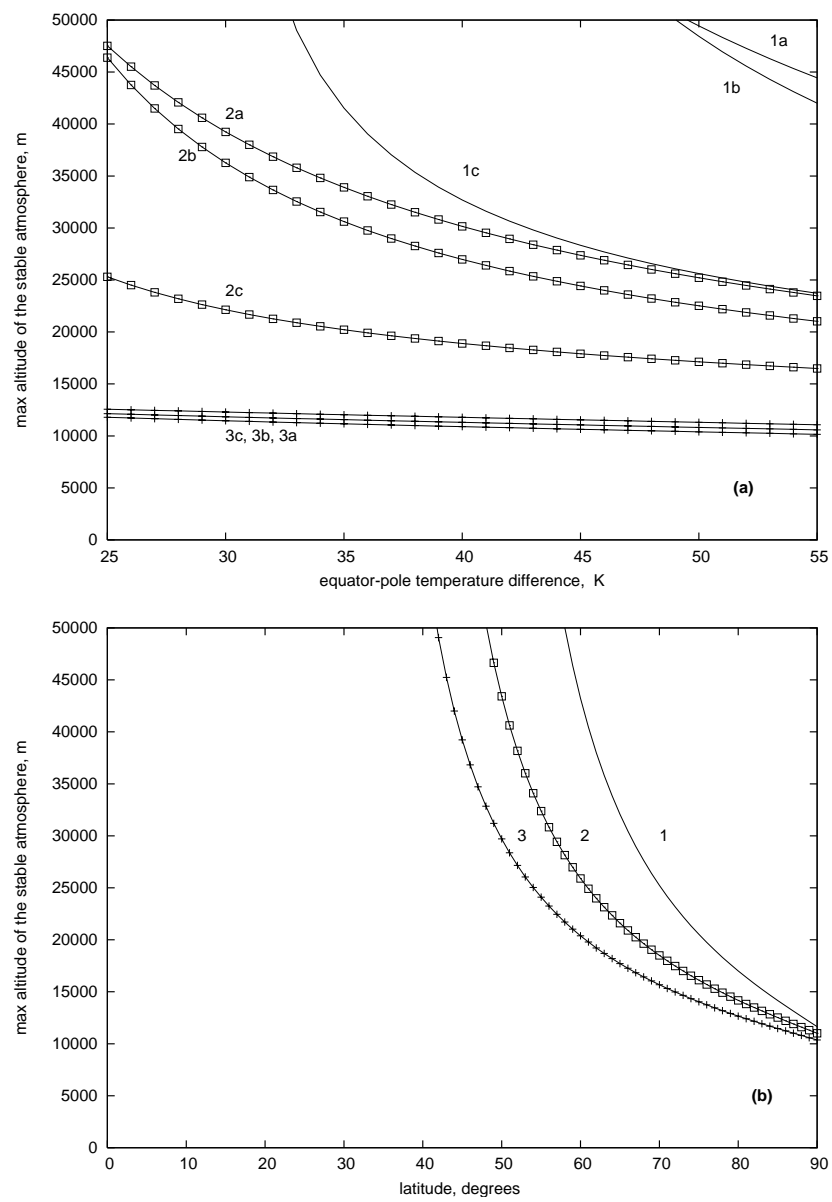

Fig. 6. Dependence of $Z_{\max }$ on $\Delta T$ (a) and $\varphi$ (b) for the case where the meridional surface temperature distribution is described as $T=T_{p}+\Delta T \cos \varphi$. In (a) the curves marked as '1a', '1b', '1c' correspond to latitudes $45^{\circ}, 60^{\circ}, 90^{\circ}$ and $\gamma=0.003 \mathrm{~K} / \mathrm{m}$; '2a', '2b', '2c' correspond to the same latitude and $\gamma=0.006 \mathrm{~K} / \mathrm{m}$; and '3a', '3b', '3c' correspond to the same latitude and $\gamma=0.009$ $\mathrm{K} / \mathrm{m}$. In (b) $\gamma=0.006 \mathrm{~K} / \mathrm{m}$, and curves '1 -3 ' corresponds to $\Delta T=25 \mathrm{~K}, 40 \mathrm{~K}$, and $55 \mathrm{~K}$.

As expected, the area of stability in latitude and $\partial V / \partial z$ parameter space decreases as the vertical temperature gradient $\gamma$ increases. For an adiabatic lapse rate, the zonal flow can become unstable for all values of $\partial V / \partial z$, even in a finite atmosphere. The area of instability appears near the equator and extends to the poles with increasing $\gamma$.

\section{Appendix}

To prove Eq. (7) it is necessary to apply to Eq. (1) an operator $\partial / a \cos \varphi \partial \lambda$ and to Eq. (2) an operator $-\partial / a \partial \varphi$ and to add. As a result we obtain (expressing $p$ and $\rho$ through $s$ and $T$ from ideal gas entropy equation)

$$
\begin{aligned}
& \frac{d f}{d t}+\frac{f}{a \cos \varphi}\left(\frac{\partial u}{\partial \lambda}+\frac{\partial(v \cos \varphi)}{\partial \varphi}\right)= \\
& \frac{1}{a^{2} \cos \varphi \rho^{2}}\left(\frac{\partial T}{\partial \lambda} \frac{\partial s}{\partial \varphi}-\frac{\partial T}{\partial \varphi} \frac{\partial s}{\partial \lambda}\right) .
\end{aligned}
$$


Differentiating the entropy conservation Eq. (5) with respect to $z$, we have

$\frac{d}{d t}\left(\frac{\partial s}{\partial z}\right)+\frac{1}{a \cos \varphi} \frac{\partial s}{\partial \lambda} \frac{\partial u}{\partial z}+\frac{\partial s}{a \partial \varphi} \frac{\partial v}{\partial z}+\frac{\partial s}{\partial z} \frac{\partial w}{\partial z}$.

Then using equations for thermal wind

$f \frac{\partial u}{\partial z}=\frac{\partial T}{\partial z} \frac{\partial s}{a \partial \varphi}-\frac{\partial T}{a \partial \varphi} \frac{\partial s}{\partial z}$

$f \frac{\partial v}{\partial z}=-\frac{1}{a \cos \varphi} \frac{\partial T}{\partial z} \frac{\partial s}{\partial \lambda}+\frac{1}{a \cos \varphi} \frac{\partial T}{\partial \lambda} \frac{\partial s}{\partial z}$

and equation for vertical gradient of entropy

$$
\frac{\partial s}{\partial z}=\frac{c_{p}}{T}\left(\frac{g}{c_{p}}+\frac{\partial T}{\partial z}\right)
$$

and adding Eq. (30) divided by $f$ and Eq. (31) divided by $\partial s / \partial z$ and subtracting the continuity Eq. (4) divided by $\rho$ we come to the equation

$$
\frac{d}{d t} \frac{f \frac{\partial s}{\partial z}}{\rho}=0
$$

Acknowledgements. The financial support for this research by the Brazilian National Council for Development of Science and Technology (CNPq) is gratefully acknowledged. I want also to thank M. V. Kurgansky and S. Nilo Figueroa for the useful discussions.

Edited by: A. Osborne

Reviewed by: two referees

\section{References}

Arnol'd, V. I.: On the conditions for nonlinear stability of stationary plane curvilinear flows of an ideal fluid (in Russian), Dokl. Akad. Nauk SSSR, 162, 975-978, 1965, (English transl.: Sov. Math., 6, 773-777, 1965.)

Baines, P. G.: The stability of planetary waves on a sphere. J. Fluid Mech, 73, 2, 193-214, 1976.

Barnes, R. T. H., Hide, R., F. R. S., White, A. A., and Wilson, C. A.: Atmospheric angular momentum fluctuations, length-of-day changes and polar motion, Proc. R. Soc. Lond, A 387, 31-73, 1983.

Bengtsson, L.: Numerical prediction of atmospheric blocking - a case study, Tellus, 33, 19-42, 1981.

Burger, A.: Scale consideration of planetary motions of the atmosphere. Tellus, 10, 2, 195-205, 1958.

Charney, J. G.: Planetary fluid dynamics. In Dynamical Meteorology, edited by Morel, P., 211-218, 1973.

Charney, J. G. and Drazin, P.: Propagation of planetary scale disturbances from the lower into the upper atmosphere, J. Geophys. Res.,66, 1, 83-110, 1961.

Chetaev, N. G.: Ustoichivost' dvizheniya. (Stability of motion), Moskva, Nauka, Glavnaya redaktsiya fiziko-matematicheskoy literatury, 1990.

James, I. N. and James, P. N.: Spatial structure of ultra-low frequency variability of the flow in a simple atmospheric circulation model. Q.J.R. Meteorol. Soc., 118, 1211-1233, 1992.
Dikiy, L. A.: Nonlinear theory of hydrodynamic instability(in Russian). Izv. Acad. Nauk SSSR, Applied mathematics and mechanics, 29, 852-855, 1965.

Dikiy, L. A. and Kurgansky, M. V.: Integral conservation law for disturbances of zonal flow and his application to study of stability (in Russian), Izv. Acad. Nauk SSSR, Fiz. Atmos. Okeana, 7, 9, 939-945, 1971.

Kurbatkin, G. P.: Investigation of ultralong atmospheric waves, in "Numerical methods for solving weather forecast and general atmospheric circulation problems". Novosibirsk, VTS SO AN SSSR, 174-226, 1970

Kurgansky, M. V.: Introduction to large scale atmospheric dynamics (Adiabatic invariants and how to use them) (in Russian), Hidrometeoizdat, Saint Petersburg, 1993.

Kurgansky, M. V., Dethloff, K., Pisnichenko, I. A., Gernandt, H., Chmielewski, F.-M., and Jansen, W.: Long-term climate variability in a simple, nonlinear atmospheric model. J. Geophys. Res., 101, D2, 4299-4314, 1996.

Lynch P.: Baroclinic instability of ultra-long waves modelled by planetary geostrophic equations. Geophys. Astrophys Fluid Dynamics, 13, 107-124, 1979.

Lynch, P., The slow equations. Quart. J. Roy. Meteorol. Soc., 115, 201-219, 1989.

McIntyre, M. E. and Shepherd, T. G.: An exact local conservation theorem for finite-amplitude disturbances to non-parallel shear flows, with remarks on Hamiltonian structure and on Arnol'd's stability theorems, J.Fluid Mech., 181, 527-565, 1987.

McNulty, R.: Vertical energy flux in planetary scale waves. Observational results, J. Atmos. Sci., 33, 7, 1172-1183, 1976.

Mu, M. and Shepherd, T. G.: Nonlinear Stability of Eady's Model, J. Atmos. Sci., 51, 23, 3427-3436, 1994.

Phillips, N.: Geostrophic motion. Rev. Geophys.,1, 2, 123-176, 1963.

Pisnichenko, I. A.: Dynamics of ultralong waves in twodimensional baroclinic atmospheric model(in Russian), Izv. Acad. Nauk SSSR, Fiz. Atmos. Okeana,16, 9, 883-892,1980.

Pisnichenko, I. A.: Influence variable static stability on the dynamics of ultralong waves in two-dimensional baroclinic model of the atmosphere, Izv. Acad. Nauk SSSR, Fiz. Atmos. Okeana, 19, 11, 1223-1226, 1983.

Pisnichenko, I. A.: A simple model of the influence of orography on behaviour of angular momentum vector components. Vortex dynamics and energetics of atmosphere investigations and climate problem, Edited by Nikiforov, E. G. and Romanov, V. F., Leningrad, Gidrometeoizdat, 219-226, 1990.

Pratt, R. and Wallace, J.: Zonal propagation characteristics of largescale fluctuations in the mid-latitude troposphere. J. Atmos. Sci. 33, 7, 1184-1193, 1976.

Shepherd, T. G.: Nonlinear saturation of baroclinic instability. Part II: Continuously stratified fluid. J. Atmos. Sci., 46, 888-907, 1989.

Starr, V. P.: Physics of negative viscosity phenomena, McGrawHill, New York, 256, 1968.

Wiin-Nielsen, A.: A preliminary study of the dynamics of transient planetary waves in the atmosphere, Tellus, 13, 3, 320-337, 1961.

Yongming, L. and Mu, M.: Nonlinear stability theorem for Eady's model of quasi-geostrophic baroclinic flow, J. Atmos. Sci., 53, 1459-1463, 1996. 